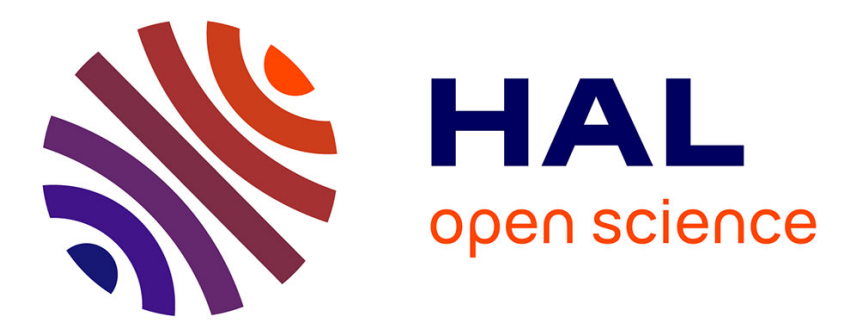

\title{
Optimizing the laser absorption technique for quantification of caesium densities in negative hydrogen ion sources \\ U Fantz, C Wimmer
}

\section{- To cite this version:}

U Fantz, C Wimmer. Optimizing the laser absorption technique for quantification of caesium densities in negative hydrogen ion sources. Journal of Physics D: Applied Physics, 2011, 44 (33), pp.335202. 10.1088/0022-3727/44/33/335202 . hal-00644267

\section{HAL Id: hal-00644267 https://hal.science/hal-00644267}

Submitted on 24 Nov 2011

HAL is a multi-disciplinary open access archive for the deposit and dissemination of scientific research documents, whether they are published or not. The documents may come from teaching and research institutions in France or abroad, or from public or private research centers.
L'archive ouverte pluridisciplinaire HAL, est destinée au dépôt et à la diffusion de documents scientifiques de niveau recherche, publiés ou non, émanant des établissements d'enseignement et de recherche français ou étrangers, des laboratoires publics ou privés. 


\title{
Optimizing the laser absorption technique for quantification of caesium densities in negative hydrogen ion sources
}

\author{
U Fantz $^{1,2}$ and C Wimmer ${ }^{2}$ \\ ${ }^{1}$ Max-Planck-Institut für Plasmaphysik, EURATOM Association, Boltzmannstr.2, \\ 85748 Garching, Germany \\ ${ }^{2}$ Lst. f. Experimentelle Plasmaphysik, Universität Augsburg, Universitätsstr. 1, 86135 \\ Augsburg, Germany \\ E-mail: fantz@ipp.mpg.de
}

\begin{abstract}
The performance of negative hydrogen ion sources, which rely on the formation of negative hydrogen ions on a surface with low work function, depends strongly on the caesium dynamics in the source. A quantitative measurement of the amount of caesium in the source during plasma-on and plasma-off (vacuum phase) is highly desirable. The laser absorption technique is optimized for the diagnostics of neutral caesium densities close to the extraction surface on which the negative hydrogen ions are generated. The setup has been simplified as much as possible utilizing also an automatic data evaluation for online measurements at high power $\mathrm{rf}$ sources. The setup has been tested and calibrated in a small scale laboratory experiment. The system and the analysis of the $\mathrm{D}_{2}$ caesium line at $852.1 \mathrm{~nm}$ is described in detail, including effects of line saturation and density depletion. The system is sensitive in the density range of $10^{13}-10^{17} \mathrm{~m}^{-3}$ (path length of about $15 \mathrm{~cm}$ ), allowing also for a temporal resolution of $40 \mathrm{~ms}$. First very promising results from the negative hydrogen ion source are presented, such as the increase of the caesium density due to the caesium evaporation and time traces before, during, and after the discharge indicating a strong caesium redistribution.
\end{abstract}

PACS: 29.25.Ni, 52.25.Os, 52.70.Kz

Submitted to: Journal of Physics D: Applied Physics

\section{Introduction}

The formation of negative hydrogen ions in hydrogen plasmas is strongly enhanced by introducing caesium to the plasma chamber [1,2]. The underlying process is the surface process, in which hydrogen atoms or positive hydrogen ions from the plasma are converted at a surface into negative hydrogen ions [3]. Since the conversion yield depends on the work function of the converter surface the work function is actively lowered by caesium deposition on metallic surfaces [4]. The work function of pure caesium with $2.14 \mathrm{eV}$ is only half as high as the work function of tungsten or molybdenum [5]. Furthermore, the formation of a dipole layer on the surface of the metal can lower the work function under high vacuum conditions $\left(\mathrm{p}<10^{-6} \mathrm{~Pa}\right)$ and a clean caesium layer to a value as low as $1.4-1.7 \mathrm{eV}$ at a thickness below one monolayer [6]. Due to the high chemical reactivity of caesium layers small amounts of impurities, for example the residual gas, can cause a degradation of the caesium layer and thus of the work function [7, 8]. Besides the time dependence of the work function in the vacuum phase of pulsed plasma operation, the plasma itself may modify the work function by plasma surface interaction processes and eventually decrease the thickness of the layer 
until the layer is removed. As a consequence, the stability and reproducibility of the caesium layer at the converter surface in the vacuum and the plasma phase determines the stability and the reproducibility of the negative ion current from such an ion source.

Negative hydrogen ion sources are commonly used for high energy accelerators [9], for example the front end of SNS (Spallation Neutron Source, Oak Ridge) [10] or at LANSCE (Los Alamos Neutron Science Center, Los Alamos) [11], and gain more and more relevance for the neutral beam injection systems of fusion devices $[12,13]$. According to the requirements small negative-ion sources (diameter of several $\mathrm{cm}$ ) with a mono-aperture extraction system are in operation with a high duty cycle for accelerators whereas for neutral beam systems large area sources (width up to $50 \mathrm{~cm}$, length up to 1 $\mathrm{m})$ with a multi-aperture extraction system are applied. The requirements are even more challenging for the neutral beam system of the next generation of a fusion device, namely the international fusion experiment ITER [14], being currently under construction in Cadarache, France. The large area source (90 cm width and $1.9 \mathrm{~m}$ height) has to provide a current density of $200 \mathrm{~A} / \mathrm{m}^{2} \mathrm{D}^{-}\left(280 \mathrm{~A} / \mathrm{m}^{2} \mathrm{H}^{-}\right)$ homogeneously from 1280 apertures $(0.2 \mathrm{~m}$ extraction area) and stable for 1 hour [15]. In order to meet these requirements an ambitious R\&D program has started at the Max-Planck-Institut für Plasmaphysik (IPP, Garching) several years ago [16]. Three test facilities are currently available using inductively coupled plasmas powered by an rf generator at $1 \mathrm{MHz}$ frequency and up to $140 \mathrm{~kW}$ power. The IPP prototype rf ion source has been chosen as the reference source for ITER in 2007 [17].

In both types of applications the performance of the negative ion source depends strongly on the caesium dynamics, i.e. the caesium evaporation and desorption from surfaces, which determines the formation of the caesium layer on the converter surface. Thus, monitoring of the caesium layer would be a highly desirable tool for source optimization. However, a routine and in-situ measurement of the work function of the converter surface in ion sources is not available and indirect methods to monitor the caesium dynamics have to be used instead. One example is the optical emission spectroscopy which monitors the emission of the caesium line at $852 \mathrm{~nm}$ close to the convertor surface as a measure for the caesium density in the plasma phase [18]. Quantification, however, needs knowledge of plasma parameters. Although this diagnostics is being routinely used in more and more systems, a deeper understanding of the caesium dynamics is still missing. As indicated by the experiments at the test facilities [19] and during experimental investigations in laboratory plasmas [8, 20], the caesium dynamics and thus the built-up of the caesium layer in the vacuum phase, i.e. between the pulses, determines the source performance.

Although there are several methods for caesium detection in the vacuum phase, for example the photoelectric current measurement (work function method), a surface ionisation detector (Langmuir detector), a quartz micro balance or a mass spectrometer, they cannot be easily used in combination with plasma operation and in the harsh environment of an high power $\mathrm{rf}$ ion source operating at high voltage. In addition diagnostic access to the source is also very limited.

A diagnostic method which offers direct access to the ground state density of a particle in the vacuum and in the plasma phase is absorption spectroscopy utilizing a resonant transition. This well known technique is being used in many applications using either white light absorption spectroscopy or (tunable diode) laser absorption spectroscopy; however the application to caesium in discharge chambers for caesium quantification is not well established so far. The white light absorption spectroscopy of the caesium resonance line at $852 \mathrm{~nm}$ has been already successfully applied to laboratory experiments [20], but is not suitable for routinely operating ion sources. Here, a robust and easy-to-use setup is highly desirable with automatic data acquisition and analysis for online monitoring in vacuum and plasma operation, being density sensitive over several orders of magnitude. The paper focuses on the caesium laser absorption technique and its application to ion sources. However, for comparisons with earlier measurements the white light absorption technique is presented as well. The development of the laser absorption setup at a laboratory experiment is described together 
with the features of the data analysis and the improvements achieved in comparison to the white light absorption technique. Finally the paper presents in detail the adoption of the customized system to the ion source and reports on first successful measurements.

\section{Caesium absorption}

Absorption spectroscopy is an active diagnostic method which is widely used for measuring densities of stable particles (atoms and molecules) in the gas and plasma phase and also gives access to the densities of radicals, ions and metastable states of particles in the plasma. Since the intensity of the signal depends on the Einstein coefficient for absorption $B_{k i}$ for a transition from the lower excited state $k$ into the higher excited state $i$, with $k$ as ground state, resonance lines are well suited for this diagnostics. Therefore the caesium resonance lines in the near infrared spectral range are ideal candidates for quantification of the ground state density of caesium in the vacuum and in the plasma phase. One has to keep in mind that caesium will be partly ionized in the plasma which means a caesium quantification is restricted to the neutrals only. Resonance lines of caesium ions are in the far UV range (between 70 and $93 \mathrm{~nm}$ [21]) and thus not easy accessible. Candidates for light sources with a detector are either a highly stabilized and intense white light source in combination with a spectrometer equipped with a CCD camera (e.g. [22]) or a tunable laser system with a photomultiplier (e.g. [23]). White light absorption spectroscopy is the more flexible and often cheaper method, whereas for laser absorption the laser and the detector are chosen according to the respective wavelength range with the advantage of improving the signal-to-noise ratio. In addition, the combination of a laser with an optical fibre coupling and a photodiode with an interference filter as detector offers a robust and compact setup applicable to ion sources.

\subsection{Selected caesium transition}

The ground state of caesium has a $5 p^{6} 6 \mathrm{~s}$ configuration resulting in a $6{ }^{2} \mathrm{~S}_{1 / 2}$ state. For the first excited configuration $5 p^{6} 6 p$ the fine structure results in two electronic states, the $6{ }^{2} \mathrm{P}_{1 / 2}$ and the $6{ }^{2} \mathrm{P}_{3 / 2}$ state. The resonance transitions correspond to the $\mathrm{D}_{1}$ line at $895.3 \mathrm{~nm}$ and the $\mathrm{D}_{2}$ line at $852.1 \mathrm{~nm}$ with transition probabilities for spontaneous emission $\left(A_{i k}\right)$ of $2.87 \times 10^{7} \mathrm{~s}^{-1}$ and $3.276 \times 10^{7} \mathrm{~s}^{-1}$, respectively [24]. Due to the lower wavelength, which implies a better availability of light sources and sensitivity of detectors, and the higher transition probability the $\mathrm{D}_{2}$ line is been chosen for the absorption diagnostics. In addition, this $852.1 \mathrm{~nm}$ line is also used for optical emission spectroscopy.

The nuclear spin $I=7 / 2$ results in a hyperfine structure characterised by the total angular momentum quantum number of the atom $F$. Thus, the ground state $6{ }^{2} \mathrm{~S}_{1 / 2}$ splits up into two hyperfine levels $(F=$ $3,4)$ and the $6{ }^{2} \mathrm{P}_{3 / 2}$ state into four hyperfine levels $(F=2,3,4,5)$. According to the selection rules for optical transitions, $\Delta F=0, \pm 1$, the caesium $\mathrm{D}_{2}$ line consists of six hyperfine lines. Because of the narrow energy splitting of the $6{ }^{2} \mathrm{P}_{3 / 2}$ hyperfine states $\left(\approx 8 \times 10^{-7} \mathrm{eV}\right)$ the Doppler broadening of the individual lines reflects the energy splitting of the ground state $\left(\approx 4 \times 10^{-5} \mathrm{eV}\right)$ only resulting in two peaks. An example is shown in figure 1(a) for a gas temperature of $1000 \mathrm{~K}$ which is typical for low pressure ion sources [25]. The central wavelengths of the separate hyperfine lines and their relative intensities are taken from [26] and [27], respectively. Due to the strong overlap of the three individual lines $\left(\Delta \lambda_{\mathrm{FWHM}}=1.86 \mathrm{pm}\right)$ in each of the two lines it is reasonable to use a Gaussian profile for each of the two lines $\left(\Delta \lambda_{\text {FWHM }}=1.87 \mathrm{pm}\right.$ left peak, $\left(\Delta \lambda_{\text {FWHM }}=1.91 \mathrm{pm}\right.$ right peak $)$ separated by $21.4 \mathrm{pm}$.

In case of white light absorption spectroscopy, the apparatus profile of the spectroscopic system determines the spectral resolution of the diagnostic system. The typical spectral resolutions of a $1 \mathrm{~m}$ spectrometer with a grating of 1800 grooves per $\mathrm{mm}$ is similar to the line separation resulting in a spectrum in which the two lines are barely separated. In case of tunable laser absorption, the line profile of the laser determines the spectral resolution which is in most cases orders of magnitudes below the line separation. A comparison of the absorption signals obtained with the white light 
absorption technique (spectral resolution $\Delta \lambda_{\mathrm{FWHM}}=15 \mathrm{pm}$ ) and a tunable diode laser (line profile 0.01 $\mathrm{pm}$ ) is shown in figure 1(b). As expected the line separation is only indicated in the white light absorption with a low number of data points whereas the laser absorption yields clearly two separated Doppler broadened lines.

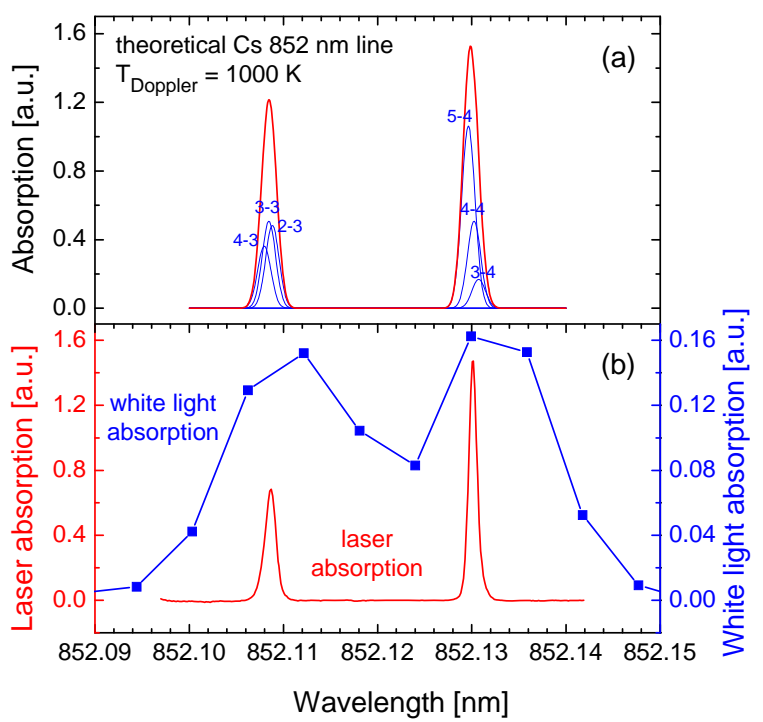

Figure 1. (a) Calculated caesium resonance line $\left(6{ }^{2} P_{3 / 2}-6{ }^{2} S_{1 / 2}\right.$ transition, $852 \mathrm{~nm}$ line $)$ in which a Dopplerprofile at $1000 \mathrm{~K}$ is used for the individual hyperfine components. (b) Absorption signals measured with laser absorption and white light absorption.

\subsection{Absorption technique}

The line absorption technique is an active diagnostic method for measuring particle densities using intensive light sources such as stabilized high-pressure lamps which emit a broad white light emission spectrum, or wavelength tunable lasers [22, 28, 29]. The method is commonly applied to atomic lines as well as to molecular spectra, from the vuv to the IR spectral range.

Under the assumption that a beam of parallel light with spectral intensity $I(\lambda, 0)$ passes through a homogeneous absorbing medium with length $\ell$ the spectral intensity $I(\lambda, \ell)$ is related to the absorption coefficient $\kappa(\lambda)$ by:

$$
I(\lambda, \ell)=I(\lambda, 0) \exp [-\kappa(\lambda) \ell] .
$$

For small values of $\kappa(\lambda) \ell$, in particular of $\kappa\left(\lambda_{0}\right) \ell$ (with $\lambda_{0}$ the central wavelength of the line), the ratio $I(\lambda, \ell) / I(\lambda, 0)$ is given by $1-\kappa(\lambda) \ell$ (linear approximation). Then, the absorption profiles of the observed spectral intensity are of the same shape and width as the spectral line profile $L_{p}(\lambda)$. At high absorption their peaks are flattened and they become broader. For the spectral line profile of a single line it is reasonable to assume a Gaussian profile caused by the Doppler broadening in the gas phase as well as in the plasma phase (however with different temperatures), as it is also reasonable for the caesium lines under consideration (see discussion above).

The wavelength-integrated absorption coefficient for transition $k \rightarrow i$, is proportional to the number of particles in lower state $k$ and simply given by:

$$
\int_{\text {line }} \kappa_{k i}(\lambda) d \lambda=\int_{\text {line }} n_{k} \frac{g_{i}}{g_{k}} \frac{\lambda_{0}^{4}}{c} \frac{A_{i k}}{8 \pi} L_{p}(\lambda) d \lambda=n_{k} \frac{g_{i}}{g_{k}} \frac{\lambda_{0}^{4}}{c} \frac{A_{i k}}{8 \pi} .
$$


$n_{k}$ is the population density of the lower level, $g_{i}$ and $g_{k}$ the statistical weights of upper and lower level, and $A_{i k}$ the Einstein transition probability for spontaneous emission. $L_{p}(\lambda)$ describes the normalised line profile (area equal to one). More precisely, $\kappa(\lambda)$ is the effective absorption coefficient, i.e. absorption minus stimulated emission. Since in most cases $n_{i}<<n_{k}$ (gases and low temperature plasmas), stimulated emission is negligible.

This means the density of the lower state of an atomic line and thus the ground state density for resonance lines is then directly accessible by

$$
n_{k}=\frac{8 \pi c}{\lambda_{0}^{4}} \frac{g_{k}}{g_{i}} \frac{1}{A_{i k} \ell} \int_{\text {line }}-\ln \left(\frac{I(\lambda, \ell)}{I(\lambda, 0)}\right) d \lambda
$$

Concerning the two experimental setups discussed above, the white light absorption spectroscopy uses a continuum source with intensity $I(\lambda, 0)$ and the corresponding spectroscopic setup determines the measured line profile given by the apparatus profile $\Delta \lambda_{\text {app }}$ which results, due to the corresponding convolution of the line profiles, in a broadening of the absorption lines. For laser absorption the line width of the tunable laser is often much smaller than the Doppler width of the spectral line such that the absorption spectrum shows the Doppler broadening directly. Since in both cases the area under the absorption line must be the same the peak intensity in the laser absorption spectrum is much higher resulting in an improved signal to noise ratio for the same ground state density and absorption length. For data evaluation, however two effects might be of relevance: one is the line saturation and the other the depopulation of the ground state density by the intense light. If not considered both lead to a nonnegligible underestimation of the ground state density.

\subsection{Line saturation}

As briefly mentioned above, the line profiles flatten for high absorption, which means line saturation occurs. Introducing $\alpha_{0}$ as the area of the absorption coefficient $\alpha_{0}=\int_{\text {line }} \kappa(\lambda) \mathrm{d} \lambda$ equation (1) reads:

$$
I(\lambda, \ell)=I(\lambda, 0) \exp \left[-\alpha_{0} L_{p}(\lambda) \ell\right] .
$$

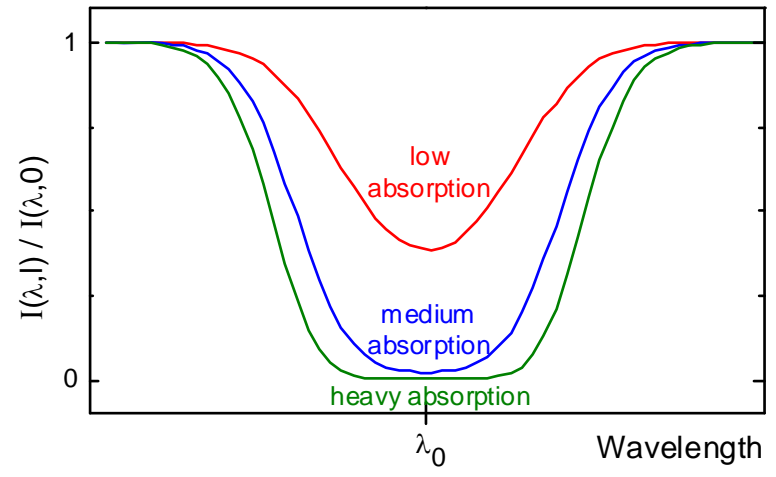

Figure 2. Examples of a line profile for three cases of line saturation.

Figure 2 shows the ratio $I(\lambda, \ell) / I(\lambda, 0)$ for different values of $\alpha_{0} \ell$ using a Gaussian line profile. As the absorption becomes stronger, the line profile becomes broader and flattens at the central wavelength, 
caused by the strong absorption of the medium. The spectral intensity of the light source is being reduced along the path length according to the absorption profile. Thus, the intensity at the central wavelength of the absorption peak is reduced first before the wings cause a reduction. After a certain path length, this results in the contrary effect. As the absorption is proportional to $I(\lambda, 0)$, the absorption at the wavelength of the wings is stronger reduced than at $\lambda_{0}$ and the line becomes broader.

\subsubsection{White light absorption}

In case of white light absorption the spectral resolution of the used spectrometers used is not good enough to identify the above features, i.e. the apparatus profile is much broader than the Doppler width according the gas temperature of the particle (see figure 1). As a consequence, the observed widths correspond almost to the apparatus profile, more precisely, to a convolution of the absorption and the apparatus profiles, and the peak absorption is strongly reduced. The apparatus profile can also be approximated by a Gaussian. In the case of small true absorption in the line centre (linear approximation), the integral absorption coefficient and the density $n_{k}$ can be determined from the measured integral absorption without knowing the line width

$$
n_{k}=\frac{8 \pi c}{\lambda_{0}^{4}} \frac{g_{k}}{g_{i}} \frac{1}{A_{i k} \ell} \int_{\text {line }} \frac{I(\lambda, 0)-I(\lambda, \ell)}{I(\lambda, 0)} d \lambda .
$$

In many cases the linear approximation no longer holds and therefore, correction factors $f_{k}$ have to be calculated relating the measured density according equation (3) to the corrected density:

$$
n_{k, \text { corr }}=f_{k} n_{k, \text { meas }} \text {. }
$$

The correction factor is then defined as the ratio of the area under the logarithm absorption profile before and after the broadening caused by the apparatus profile

$$
f_{k}=\frac{\int_{\text {line }}-\ln \left(\frac{I(\lambda, \ell)}{I(\lambda, 0)}\right) d \lambda}{\int_{\text {line }}-\ln \left(\frac{I_{\text {meas }}(\lambda, \ell)}{I(\lambda, 0)}\right) d \lambda}=\frac{\alpha_{0} \ell}{A_{\text {meas }}} .
$$

$A_{\text {meas }}$ is the area of the measured logarithm absorption signal. Thus, the correction factor depends not only on the optical thickness of the medium, i.e. $\alpha_{0} \ell$, but also on the broadening caused by the apparatus profile, i.e. the ratio of the width of the apparatus profile to the width of the Doppler profile of the absorption coefficient. Hence, the stronger the broadening of the measured profile the higher is the correction factor, achieving soon very high correction factors. For the example shown in figure 1 (15 pm apparatus profile, $1000 \mathrm{~K}$ gas temperature for the Cs line, $15 \mathrm{~cm}$ absorption length) the correction factor is approximately one for caesium densities below $2 \times 10^{15} \mathrm{~m}^{-3}$ and rises to three for $10^{16} \mathrm{~m}^{-3}$ and ten at $1.5 \times 10^{16} \mathrm{~m}^{-3}$, which means it is very sensitive on errors in the measured density.

\subsubsection{Laser absorption}

In the case of laser absorption the line width of the laser line is much smaller than the width of the absorption line. The absorption profile is resolved and line saturation is obtained directly. However the broad intrinsic baseline of the laser emission is also recorded by the photodiode and causes a line saturation effect. Although the laser line is orders of magnitude higher than the baseline this results in 
an offset of the absorption signal. This is of particular importance if the laser peak is being almost absorbed. Taking into account the baseline in the analysis, equation (4) gives

$$
I(\lambda, \ell)=I(\lambda, 0) \exp \left[-\alpha_{0} L_{p}(\lambda) \ell\right]+b I(\lambda, 0),
$$

with the offset depending on the wavelength. Similar to the procedure for the white light absorption, a correction factor (equation (6)) is introduced, being in this case defined as

$$
f_{k}=\frac{\alpha_{0} \ell}{\int_{\text {line }}-\ln \left[\exp \left(-\alpha_{0} L_{p}(\lambda) \ell\right)+b\right] d \lambda} .
$$

The result is shown in figure 3a in which the absorption signal (logarithm) is calculated with and without an offset $(b=0.05)$. Since the determination of the offset is not straightforward and very sensitive on the signal-to-noise ratio, a fitting procedure is been used instead. This is illustrated in figure 3(b), where the measured signal is been fitted in particular in the line wings by a Gaussian profile (Doppler profile). The correction factor is typically between one and three for the parameters mentioned above ( $1000 \mathrm{~K}$ Doppler broadening, absorption length $15 \mathrm{~cm}$ ) for caesium densities $1 \times 10^{16}$ $\mathrm{m}^{-3}\left(f_{k}=1.1\right)$ and $3 \times 10^{16} \mathrm{~m}^{-3}\left(f_{k}=2.8\right)$, and is taken onto account in the results presented in this paper.
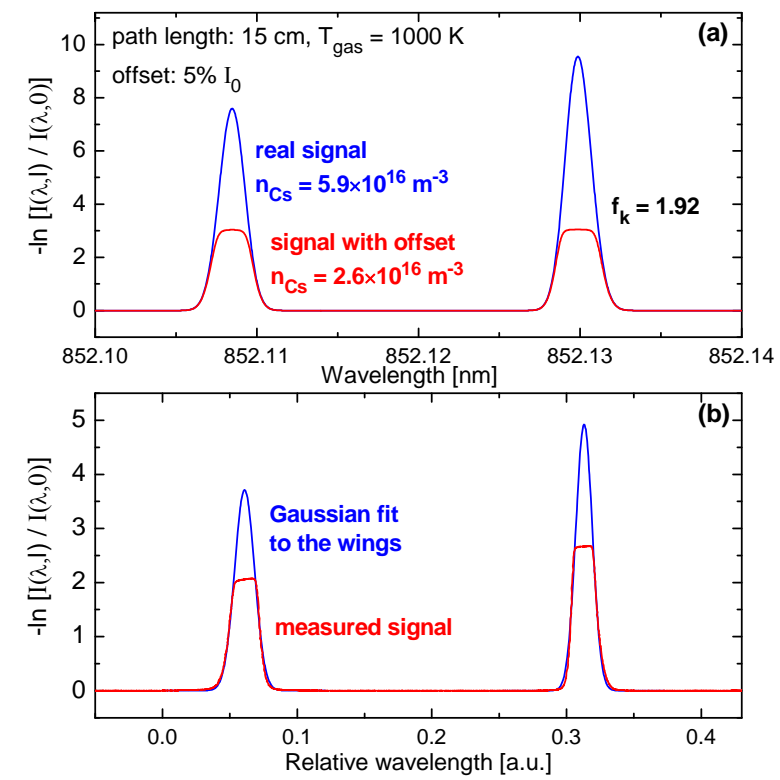

Figure 3. (a) Absorption signal calculated with and without an offset $(b=0.05)$. (b) Measured absorption signal fitted to the wings of the line profile by a Gaussian profile.

\subsection{Depopulation of the ground state density}

The interaction of the intense and narrow band laser light with the absorbing particles at the corresponding velocity along the line-of-sight reduces the population of particles in the ground state and enhances the population in the upper state. Thus, a "Bennet hole" is created in the velocitydependent population distribution, which has a Gaussian population distribution in the undisturbed case. Since the laser light is wavelength-tuned in a time scale (typically below milliseconds) longer 
than the relaxation (lifetime $<10^{-7} \mathrm{~s}$ ), the system is in equilibrium. However, the wavelength dependence on the absorption coefficient has to be taken into account, resulting in a reduction of the absorption coefficient by a constant factor [30]. As a result the measured absorption signal is being reduced; the depopulation however is not to be recognized in the absorption profile.

In order to test the relevance of such an effect, the laser light can be attenuated by neutral density filters: with increasing attenuation the absorption signal saturates, as described in [31].

\section{Customized laser absorption}

The laser absorption setup has been customized for routine and robust online measurements of the caesium line absorption at the ion source test facility. Specifications of the individual components as well as the controlling circuit and the data acquisition system are described in the following.

\subsection{Setup}

A tunable mode hop free, single mode distributed feedback (DFB) diode laser system with a total tunable range of $851 \mathrm{~nm}-853 \mathrm{~nm}$, a spectral line width of $0.01 \mathrm{pm}$ and a maximum output power of $150 \mathrm{~mW}$ is used. The system is taken from Sacher Lasertechnik $\mathrm{GmbH}$ and consists of a laser head (CHEETAH DFB-852-0609-0078) and a laser controller (Pilot PC 500); the technical specifications are summarized in table 1 . The laser beam has a diameter of approximately $1 \mathrm{~mm}$. The wavelength of the diode laser is set into the region of the caesium $\mathrm{D}_{2}$ line by temperature modulation. Tuning over the caesium $\mathrm{D}_{2}$ line is done by current modulation, which is generated linear to an applied modulation voltage by the diode laser controller.

Table 1. Technical data of the DFB diode laser.

\begin{tabular}{ll}
\hline Parameter & Value \\
\hline Tunable range & $851 \mathrm{~nm}-853 \mathrm{~nm}$ \\
Maximum output power & $>140 \mathrm{~mW} @ 852.0 \mathrm{~nm}$ \\
$\begin{array}{l}\text { Line width } \Delta \lambda_{\text {FWHM }} \\
\text { Wavelength tuning by } \\
\text { temperature modulation }\end{array}$ & $50.01 \mathrm{pm}$ \\
$\begin{array}{l}\text { Resolution of temperature } \\
\text { set-point }\end{array}$ & $1 \mathrm{kK}$ \\
$\begin{array}{l}\text { Wavelength tuning by } \\
\text { current modulation } \\
\text { Maximum current } \\
\text { Resolution of current tuning }\end{array}$ & $3 \mathrm{pm} / \mathrm{mA}$ \\
& $200 \mathrm{~mA}$ \\
& analog by applied \\
\hline
\end{tabular}

For detection a Si-PIN photodiode from Laser Components GmbH (PDSIU500FC12-T-O) has been chosen which has an active area of $0.5 \times 0.5 \mathrm{~mm}^{2}$. In order to avoid an offset in the detected current of the photodiode during plasma operation, an interference filter $\left(\lambda_{\text {centre }}=852 \mathrm{~nm}, \Delta \lambda_{\text {FWHM }}=10 \mathrm{~nm}\right)$ is used. Tests show that the photodiode does not react on the caesium line emission in the plasma phase which means the same line in plasma emission has not to be taken into account into the absorption measurement. The current of the photodiode is amplified by a transimpedance amplifier (Thorlabs PDA 200). The voltage is recorded together with the corresponding modulation voltage as indicated in figure 4. 
The light of the diode laser is coupled into an optical fibre with a core diameter of $105 \mu \mathrm{m}$. A lens with a focal length of $25.4 \mathrm{~mm}$ is used to create a beam diameter of $11.5 \mathrm{~mm}$. In order to minimize the effect of depopulation of the ground state by the intense laser light the laser can be attenuated by using a neutral density filter (as justified later on, a transmission of $1.8 \%$ at $852 \mathrm{~nm}$ is being used in the present setup). Behind the absorption medium the light is coupled by a lens with the same focal length into an optical fibre ending at the photodiode as detector. A wider fibre with a core diameter of 200 $\mu \mathrm{m}$ is used to enhance the coupling efficiency decreased by the optical aberration of the lens. (The individual components of the light path are to be seen in figure 7.)

\subsection{Controlling and timing}

Figure 4 shows a schematic diagram of the individual components of the laser absorption setup. The photodiode amplifier has an amplification of $5 \times 10^{2}$ to $5 \times 10^{7} \mathrm{~V} / \mathrm{A}$, determining also the temporal resolution of a measurement: the higher the amplification the slower the measurement.

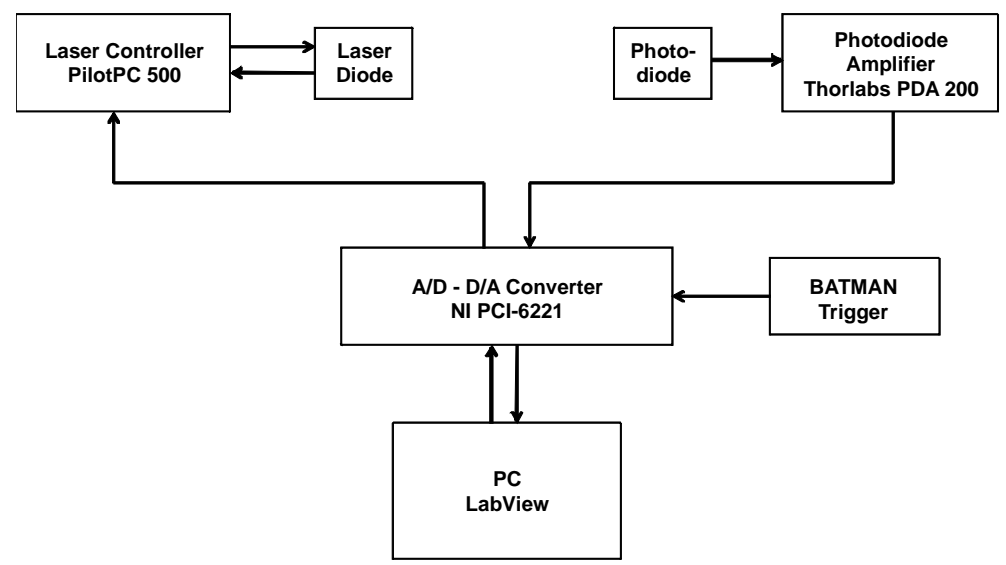

Figure 4. Schematic diagram of the controlling circuit and data acquisition system.

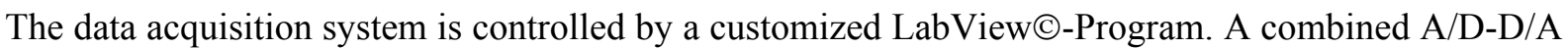
converter is used to generate voltage ramps for the wavelength modulation recording simultaneously the photodiode signal. In order to enhance the signal-to-noise ratio, averaging of spectra is provided. As indicated above, the frequency of the voltage ramp and thus the temporal resolution is a trade-off between the attenuation of the laser light and the amplification factor of the photodiode since a slow amplifier determines the temporal resolution. Investigations showed, that an amplification $5 \times 10^{5} \mathrm{~V} / \mathrm{A}$ is compatible with a frequency of $25 \mathrm{~Hz}$. In order to use the full dynamic range of the amplifier and the A/D converter a voltage of several $\mathrm{V}$ is desirable which means in turn the photodiode current should be in the range of several $\mu \mathrm{A}$. Hence, the neutral density filter should be chosen accordingly.

For the evaluation of the laser absorption spectrum a wavelength calibration is needed. Since there is no wavelength sensitive element in this simple setup, the wavelength calibration can only be done by using the wavelength positions of the two resolvable Doppler-broadened peaks assuming linear wavelength modulation with the applied diode laser current. The latter is a good approximation for a mode hop free diode laser.

For the wavelength scan a range of approx. $40 \mathrm{pm}$ is been chosen which allows for obtaining the two peaks with their separation of $21.4 \mathrm{pm}$ and the baseline. This results together with a recording frequency of 25 spectra per seconds in a scan velocity of $1 \mathrm{~nm} / \mathrm{s}$.

As indicated in figure 4, the data acquisition system can be coupled with the trigger from the experiment which is of particular importance for the pulsed mode operation of the ion source at the 
test facility BATMAN. A delay and the total recording time can be adjusted according to the experimental needs. Typically, 2 to 5 spectra are averaged resulting in a 5 to $12.5 \mathrm{~Hz}$ time resolution.

\subsection{Data analysis}

For the first tests, the spectra have been analysed manually according equation (3). However for online monitoring of signals an automatic analysis routine is highly desirable. The routine, implemented in the LabView $@-$-Program, integrates the (optionally averaged) spectrum using equation (3) and saves the value together with the time which allows for recording of a long time interval. The spectrum without absorbing medium, i.e. $I(\lambda, 0)$, is been measured before the measurement campaign and stored as baseline spectrum. In order to minimize the influence of drifts in the reference spectrum $I(\lambda, 0)$, for example due to window coatings or misalignments an automatic baseline fitting procedure will be implemented in future and is not applied to the measurements shown here.

Corrections factors which might be necessary due to line saturation effects or due to depopulation are not implemented in the routine analysis since this requires a careful manual analysis of the spectra. However, a fitting procedure to correct for weak line saturation caused by the offset is optional.

The ground state density represents clearly the total caesium density in the vacuum phase, whereas in the plasma the population of the excited states has to be taken into account. Since low pressure plasmas are far from thermal equilibrium the population of the excited states is far from a Boltzmann distribution, which means their population do not contribute significantly to the total neutral caesium density. Thus the ground state density $n_{k}$ is considered as the total neutral caesium density $n_{C s}$.

A comparison of results from the automatic routine with the manual data evaluation resulted in a very good agreement with deviations of less than $10 \%$ over two orders of magnitude in the signal and the whole time interval of $200 \mathrm{~min}$ in which caesium has been evaporated from a caesium dispenser [32].

\section{Calibration at the caesium reference cell}

For the very first investigations a commercially available caesium absorption cell has been used which allows testing of the laser absorption system as well as the analysis procedure. In addition, the effect of depopulation is been analysed systematically allowing for an identification of the strength of the required neutral density filters and a measurement of the corresponding correction factors. In a similar manner the line saturation is investigated as well.

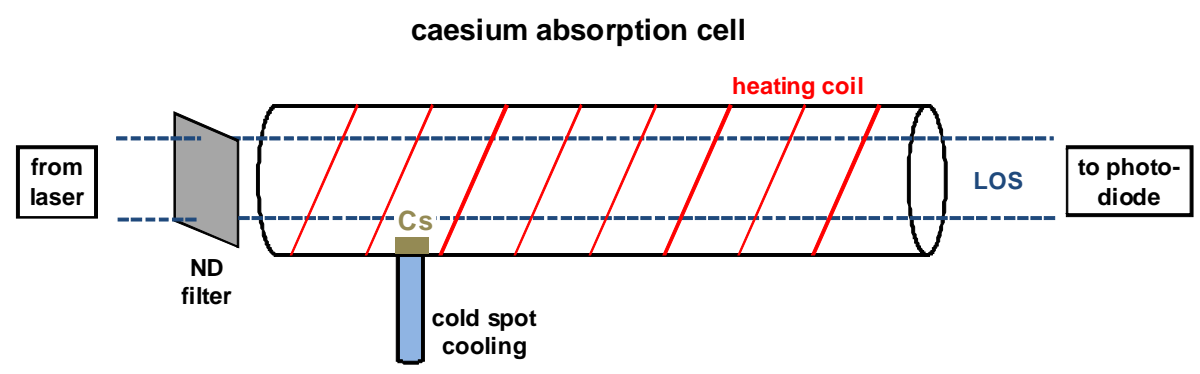

Figure 5. Experimental setup for the laser absorption at the caesium absorption cell.

Figure 5 shows a sketch of the experimental setup in which the line-of-sight of the fibre optics is only indicated as this is shown in detail in figure 7. The closed absorption cell has a length of $75 \mathrm{~mm}$ and a diameter of $19 \mathrm{~mm}$. Heating of the cell (up to about $80^{\circ} \mathrm{C}$ by heating wires) and cooling of a small point of the surface (cold spot) to a definite temperature, results in a well controlled vapour pressure. The cold spot control was achieved by an in-house development using a copper cylinder $(6 \mathrm{~mm}$ diameter) to contact the cell and which is temperature controlled by a thermoelectric cooler. The 
temperature of the cell is set to be more than $10^{\circ} \mathrm{C}$ above the cold spot temperature $\left(T_{c-s}\right)$ and determines the gas temperature $\left(T_{\text {gas }}\right)$. The caesium density is then given by: $n_{C s}=p_{\text {vapour }}\left(T_{c-s}\right) /\left(k_{B} T_{\text {gas }}\right)$ using the caesium vapour pressure curve [33]. The error of the temperature measurement is determined to be $\pm 1^{\circ} \mathrm{C}$. The cold spot temperature is varied between $-20^{\circ} \mathrm{C}$ and $+30^{\circ} \mathrm{C}$ allowing for investigations of caesium densities from $10^{14}$ up to $10^{17} \mathrm{~m}^{-3}$.

The measured caesium density can be compared with the actual density for different laser intensities (different neutral density (ND) filters) and for different vapour pressures (different cold spot temperatures). For the determination of the ground state depopulation, the depletion of the individual hyperfine structure lines has to be taken into account [31]. Since for the analysis the integral of all hyperfine components is used it is sufficient to obtain the correction for the full $\mathrm{D}_{2}$ line.

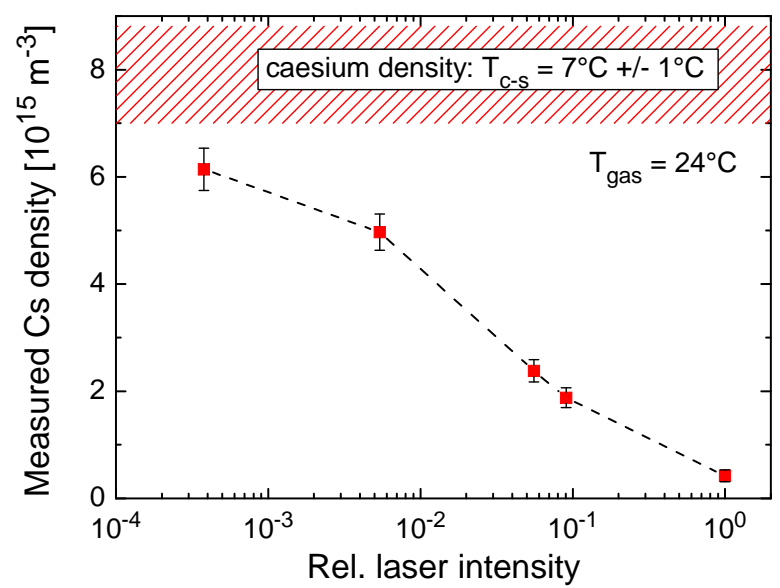

Figure 6. Caesium density measured by laser absorption as a function of the laser intensity. The dashed area indicates the density according the caesium vapour pressure.

The results for the caesium densities at fixed cold spot temperature and fixed gas temperature of the absorption cell are plotted in figure 6. The dashed region represents the caesium density according to the vapour pressure with the above mentioned uncertainty of $1^{\circ} \mathrm{C}$. Depending on the attenuation of the laser intensity, i.e. strength of the neutral density filter, the depletion and thus the correction factor decreases. Obviously an attenuation of more than three orders of magnitude is required to neglect depopulation effects. On contrary, the amplification of the photodiode has to be increased with consequences on the temporal resolution, as discussed above. In order to achieve an acceptable temporal resolution a neutral density filter with a transmission of $1.8 \%$ at $852 \mathrm{~nm}$ is been chosen. It has to be taken into account that the correction factor also depends on the value of the absorption coefficient, so the real caesium density and the scaling with the absorption length must be considered as well. These dependencies generate a large uncertainty in the correction factors. On account of this, for the present investigations, which means demonstration of the applicability of the laser absorption to negative ion sources, this correction has not been taken into account and all values represented here give the lower limit for the caesium density.

The investigations on the correction for the line saturation effect resulted in a very good representation of the caesium density by using the above mentioned fitting procedure, i.e. the Gaussian fit to the wings of the lines. It turned out that the effect appears at densities above $2 \times 10^{16} \mathrm{~m}^{-3}$ increasing the correction factor almost exponentially to four at measured densities of $4 \times 10^{16} \mathrm{~m}^{-3}$ and thus a true density above $10^{17} \mathrm{~m}^{-3}$ for the absorption length of $75 \mathrm{~mm}$. Since the fitting becomes more and more uncertain for these high values, this represents the upper detection limit. 


\section{Proof-of-principle measurements and results from the lab}

\subsection{The planar ICP}

The complete experimental setup has been applied first to a laboratory experiment which provides a good access and can be equipped simultaneously with other caesium diagnostics as well as with different caesium supplies [20].

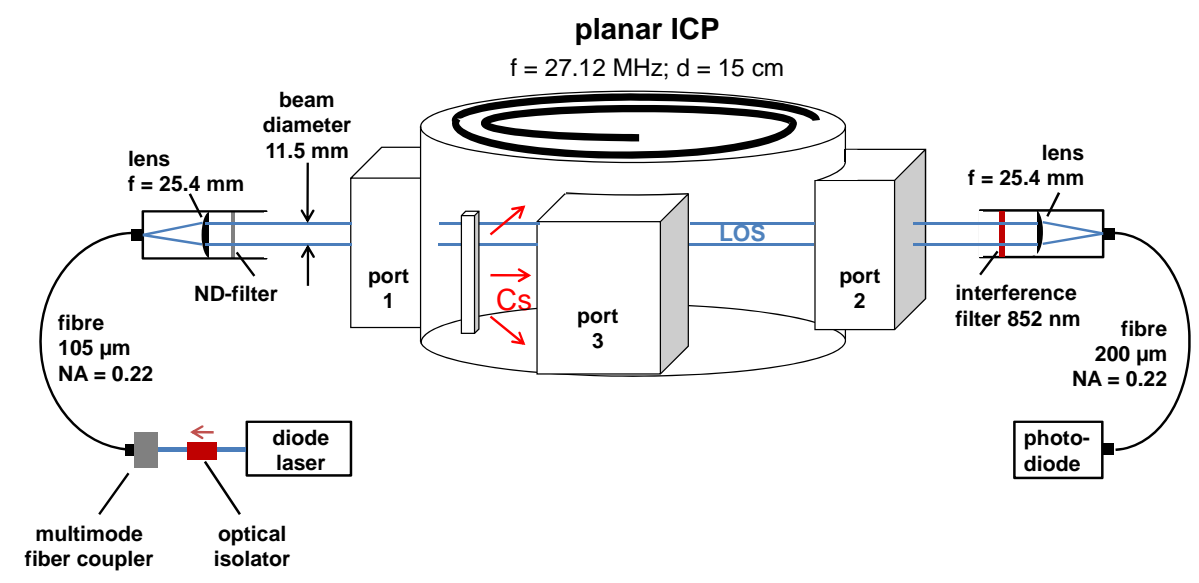

Figure 7. Experimental setup for the laser absorption at the planar inductively coupled plasma (ICP).

The plasma is generated by inductively coupling (ICP) using a planar coil with a Faraday screen at the top of the stainless steel cylinder $(15 \mathrm{~cm}$ in diameter, $10 \mathrm{~cm}$ in height). The rf generator has a maximum output power of $600 \mathrm{~W}(\mathrm{f}=27.12 \mathrm{MHz}$ ). A matchbox is used to adjust the plasma impedance. As shown in figure 7 the chamber is equipped with several diagnostic ports, the bottom plate houses several feedthroughs and ports as well. Hydrogen plasmas can be generated in the pressure range of $1 \mathrm{~Pa}$ to $50 \mathrm{~Pa}$. Mixtures with helium are often used to measure the electron temperature by optical emission spectroscopy and to influence the electron temperature and density actively. Typical parameters of hydrogen plasmas at $10 \mathrm{~Pa}$ are electron densities of $10^{16} \mathrm{~m}^{-3}$ and electron temperatures of $2 \mathrm{eV}$, measured by Langmuir probes and optical emission spectroscopy [34].

The inductively coupled plasma source operates at similar vacuum conditions and plasma parameters as the ion sources. Similar caesium evaporation rates are used as well using either caesium dispenser from SAES [35] which contain $\mathrm{Cs}_{2} \mathrm{CrO}_{4}$ embedded in $\mathrm{Al}$ and $\mathrm{Zr}$ acting as oxidant or the standard caesium oven of the ion sources at IPP (liquid caesium reservoir) as utilized at the IPP test facilities. From the optical emission spectroscopy of the caesium line at $852 \mathrm{~nm}$ it is known that the amount of caesium evaporated is in the similar density range [20].

The experimental setup for the laser absorption is shown in figure 7, showing also the line-of-sight and the geometry for the caesium dispenser with the typical amount of $10 \mathrm{mg}$ mounted at the bottom plate. Alternatively to the laser absorption setup the white light absorption spectroscopy can be installed at the same ports. For the latter, a stabilized high-pressure xenon arc lamp (OSRAM XBO 250W) acts as light source and a $1-\mathrm{m}$ spectrometer with a CCD detector (apparatus profile (Gaussian) $\Delta \lambda_{\text {FWHM }}=15$ $\mathrm{pm}$ ) and fibre optics are used for the measurements. Typically five spectra are averaged allowing for a recording time of two data points per minute.

\subsection{Vacuum measurements}

Figure 8 shows the absorption lines close to the detection limit for both setups. As expected a much 
better spectral resolution and signal-to-noise ratio is obtained for the laser absorption. For the absorption length of $15 \mathrm{~cm}$ the detection limit for the caesium density $\left(\mathrm{n}_{\mathrm{Cs}}\right)$ is at $3.5 \times 10^{13} \mathrm{~m}^{-3}$ which means a factor of 40 better than the white light absorption. In addition the dynamic range is also enlarged, since line saturation effects result in smaller correction factors and thus uncertainties at same caesium densities than for white light absorption. During plasma operation however the rf-noise can influence the signal-to-noise ratio resulting in a change of the lower detection limit. Thus, a density range of typically $5 \times 10^{13} \mathrm{~m}^{-3}$ to $10^{17} \mathrm{~m}^{-3}$ (limited by the line saturation effect) is accessible, being perfectly in the range in which the ion sources operate.
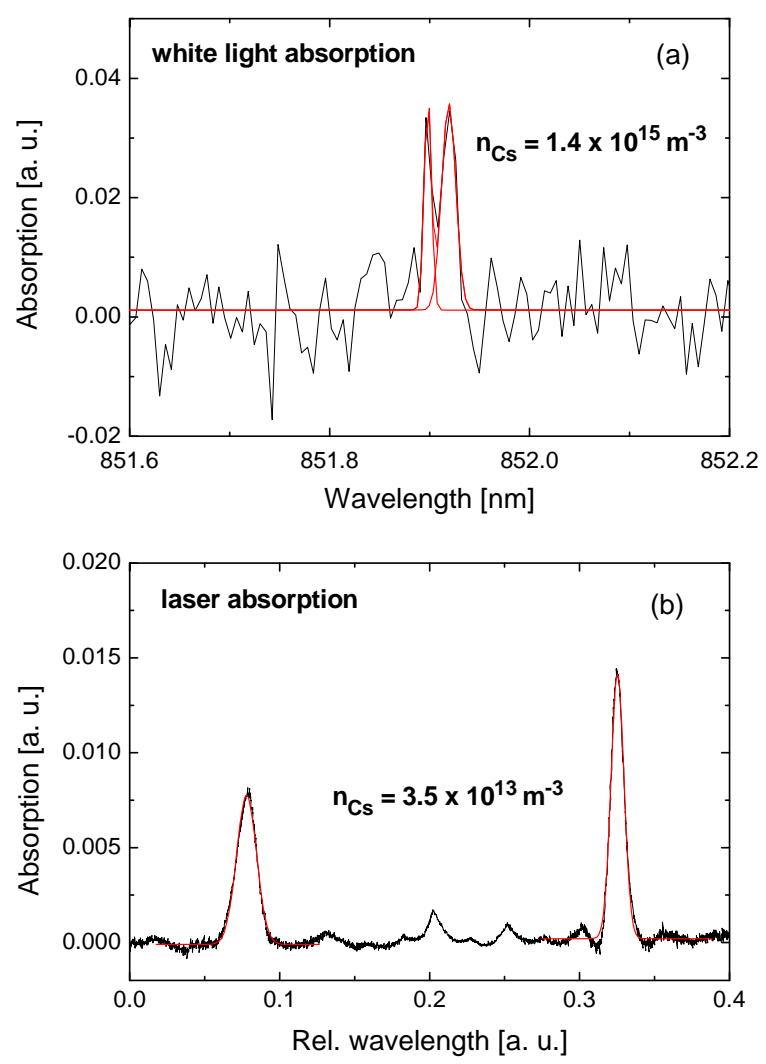

Figure 8. Absorption signal for white light absorption (a) and laser absorption (b) at the detection limit. The Caesium lines are fitted by a Gaussian profile.

A good long term stability of the laser absorption system and a very good agreement of the automatic data analysis with the manual analysis have been demonstrated [32] by using a $100 \mathrm{mg}$ caesium dispenser from Alvatec [36].

The response of the laser absorption during a dynamic change of the signal and thus the recording of time traces has been checked by a comparison with the signal of the surface ionization detector (SID). The SID measures the caesium ionized at a hot tungsten surface by applying a voltage between the outer spiral wire and the inner wire [20]. The SID is mounted on the bottom plate in line-of-sight of the caesium dispenser. The measurements were taken at a background vacuum pressure of $10^{-5}$ mbar. As shown in figure 9(a) a very good agreement between the two methods is obtained keeping in mind that they measure at different locations with different detection geometries. At $t=0$ the caesium dispenser has been switched on (after being stopped a certain time to achieve a certain dynamic range 
in caesium density) and both signals follow the abrupt caesium enhancement and the slow decay of the signal. This is the typical behaviour of the used SAES caesium dispenser evaporating at a constant heating current. Since the SID can only detect relative changes of the caesium flow, the scaling of the axes is set to match the maximum of the caesium density measured by the laser absorption spectroscopy. Line saturation effects have been taken into account being obviously relevant for reflecting the peak as the comparison of the corrected with the uncorrected signal demonstrates.
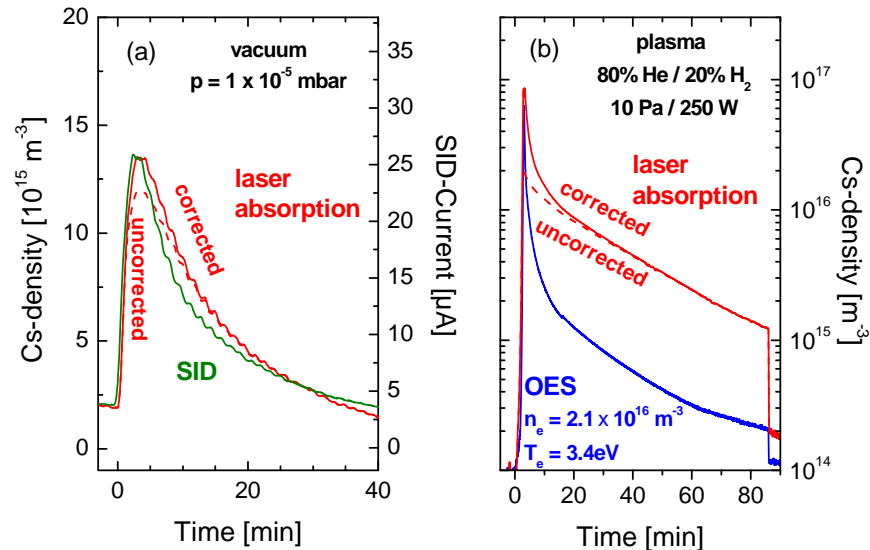

Figure 9. Time traces of the caesium density determined by laser absorption compared to the signal obtained by the surface ionization detector (SID) in the vacuum phase (a) and by optical emission spectroscopy in the plasma phase (b).

\subsection{Plasma measurements}

In case of plasma operation the laser absorption signal is compared with the signal from the optical emission spectroscopy (OES) as SID measurements are not compatible with plasma operation. For simultaneous measurements a line-of-sight perpendicular to one of the laser absorption but in the same plane is been chosen. Such differences in the geometry are of no relevance for the comparison because caesium is distributed homogenously in the chamber by the plasma. Since the spectroscopic system is intensity calibrated caesium densities can be deduced from the line emission of Cs $852 \mathrm{~nm}$ line provided the electron density and temperature are known [18], Measurements of these parameters have been carried out with a Langmuir probe system in the same experiment at same discharge parameters, but without evaporation of caesium. Caesium however will change the plasma parameters depending on the amount of caesium in the discharge. Therefore it has to be kept in mind that the OES data of figure $9 b$ are obtained using the $n_{e}$ and $T_{e}$ values of the pure $H / H e$ discharge.

Due to the low ionization energy of caesium $(3.89 \mathrm{eV})$ caesium is easily ionized in the plasma. The next ionization stage needs much higher energies (about $22 \mathrm{eV}$ ), which means the probability for doubly ionized caesium in low temperature plasmas is rather low. It has to be kept in mind that laser absorption and OES give access to the caesium density of the neutral caesium and that the values obtained in the plasma phase do not account for the ions and thus do not represent the total caesium amount in the chamber.

Figure 9(b) compares the caesium densities from both diagnostic methods over a dynamic range of three orders of magnitude. As for figure 9(a) two curves are given for the laser absorption accounting for the line saturation effect (corrected) or not (uncorrected). The $\mathrm{H}_{2} / \mathrm{He}$ plasma at $10 \mathrm{~Pa}$ pressure has been switched on first and at $\mathrm{t}=0$ the caesium evaporation from a caesium dispenser has been started. The caesium density increases instantaneously followed by a decrease; a similar behaviour as obtained in the vacuum case but the dynamic range is much higher in plasma operation. The signals are in 
reasonable agreement for the peak value (at $t \approx 0$ ), the OES densities falling more rapidly than the results from the laser absorption. As mentioned above OES relies on the knowledge of electron density and temperature. Constant values have been used for the time traces representing values for Caesium free discharges. The huge caesium amount however might change the plasma parameters which cannot be accounted for in the analysis. Since the analysis of the laser absorption is independent on plasma parameters the more reliable method is the laser absorption. Even if line saturation or depopulation effects would have been neglected the results represent a lower limit for the caesium density.

\section{Start of operation at the negative ion source}

Since the preceding investigations demonstrated the capabilities of the diagnostic system with respect to reliability and reproducibility, dynamic range, temporal resolution and analysis procedure, the laser absorption setup has been applied to the IPP prototype rf ion source at the test facility BATMAN (BAvarian Test MAchine for Negative ions).

\subsection{The BATMAN rf source}

The IPP prototype rf ion source can be basically divided into three parts: the driver, the expansion chamber and the extraction region [9]. The hydrogen plasma is generated by inductively coupling in a cylindrical driver ( $24.5 \mathrm{~cm}$ diameter, $15 \mathrm{~cm}$ length) surrounded by a water-cooled $\mathrm{rf}$ coil connected to a $140 \mathrm{~kW}, 1 \mathrm{MHz}$ oscillator. The plasma expands into a rectangular expansion chamber (copper coated stainless steel chamber with the width $\times$ height $\times$ depth of $32 \times 59 \times 23 \mathrm{~cm}^{3}$ ) which represents the source body and houses permanent magnets for the generation of a magnetic field parallel to the extraction system. This filter field with a typical strength of $7 \mathrm{mT}$ cools the electrons down and reduces the amount of the co-extracted electrons. The plasma in this extraction region has electron densities in the range of $10^{17} \mathrm{~m}^{-3}$ and temperatures of $1 \mathrm{eV}[37,38]$. The multi-aperture grid system consists of three grids: the plasma grid which is on source potential, the extraction grid with permanent magnets to remove the co-extracted electrons out of the beam, and the grounded grid. Typical extraction voltages are between 8 and $10 \mathrm{kV}$, total acceleration voltages are around $20 \mathrm{kV}$.

The standard IPP caesium oven is mounted in the upper half of the backplate of the expansion chamber. The oven houses three ampoules with $1 \mathrm{~g}$ caesium each and is heated up to temperature of $200^{\circ} \mathrm{C}$. The temperature control is used to regulate the caesium release from the oven. Typical evaporation rates are $10 \mathrm{mg} / \mathrm{h}$. Based on experimental experience, caesium conditioning of the source improves if the chamber walls are kept at temperatures of typically $45^{\circ} \mathrm{C}$ and the plasma grid at $150^{\circ} \mathrm{C}$. Due to these elevated temperatures strong caesium accumulation is avoided enhancing also the redistribution of caesium. The caesium layer at the plasma grid acts as converter surface for the flux of atomic hydrogen and positive hydrogen ions from the driver. Thus, understanding and controlling the caesium dynamics in this extraction region has the highest priority in source optimisation at present.

BATMAN is the short pulse test facility with beam extraction of $4 \mathrm{~s}$ during the typical $6 \mathrm{~s}$ plasma discharge every 3 minutes and is dedicated for improving and understanding of the physical performance of the IPP prototype ion source. Thus, the ion source is well equipped with plasma diagnostic systems, as for example OES and Langmuir probes. A typical experimental campaign lasts several weeks, starting with a cleaned source, i.e. without caesium, in hydrogen at around 0.6 Pa and reduced power to limit the co-extracted electron current. This is followed by caesium conditioning of the source, which means continuous caesium evaporation from the caesium oven adjusting the oven temperature according to the source performance. Pressure and power are adjusted accordingly until the required performance is achieved: high ion current density and low co-extracted electron current at low pressure, i.e. 0.3 Pa. Parameters scans, such as magnetic filter field studies, power scan etc. are performed only at stable source conditioning. Caesium conditioning is a quite tedious task and can take several days of 100 pulses per day [16]. Deuterium operation is usually undertaken after caesium 
condition of the source in hydrogen since beam-on time is limited in deuterium to reasons of radiation protection due to neutrons generated via the D-D reaction on the calorimeter. In that case source and diagnostics are operated remotely from outside the experimental hall.

\subsection{Laser absorption at BATMAN}

The laser absorption setup, as shown in figure 7, has been mounted at BATMAN using a vertical lineof-sight which passes the source parallel to the plasma grid in vertical direction at a distance of approx. $2 \mathrm{~cm}$. The length is $60 \mathrm{~cm}$. For OES a similar line-of-sight is available which allows for a comparison of the results. The setup illustrating both lines of sight is shown in [32]. The system has been coupled to the trigger system of BATMAN. An interlock system assures save laser operation. Optical signal transmission lines have been chosen to minimize the rf noise during plasma operation. This was in particular necessary for the long connection between the laser controller (the laser is placed below the experimental hall in the cellar which results in $20 \mathrm{~m}$ length) and the D/A converter. The temporal resolution is again limited by the laser system i.e. $40 \mathrm{~ms}$ can be achieved, but using an averaging of 2 to 5 spectra data points 80 to $200 \mathrm{~ms}$ can be achieved, respectively. The neutral density filter with the transmission of 0.018 is been used to minimize the influence of the depopulation, however the extended path length - from $15 \mathrm{~cm}$ in the reference cell to $60 \mathrm{~cm}$ at the ion source - has to be accounted for, resulting in a correction factor of typically $1.5 \pm 0.5$. Since this correction is not been taking automatically into account it has to be kept in mind that all following caesium densities are underestimated by a factor in this range.

\subsection{First results}

The first systematic measurements are carried out during the caesium conditioning of the ion source directly in the beginning of an experimental day. The day before caesium has been evaporated; however reconditioning is needed every morning before reaching the same parameters as the day before. Data are taken only in the vacuum phase, i.e. between the discharges, a few seconds before the start of the plasma ignition. Figure 10 shows the growth of the caesium density one order of magnitude over a period of approximately one hour source operation which is related to the increase of the temperature of the caesium oven, one error bar of the measured caesium density is plotted as an example. The temperature is measured at the cold spot of the oven which is the temperature of the body temperature; all other temperatures of the six temperatures control units are set to be more than $20{ }^{\circ} \mathrm{C}$ higher. The cold spot determines the vapour pressure and thus the evaporation rate. Changes of the set-value of the temperature are indicated in figure 10 which shows also the stepwise increase after a few minutes, i.e. 1-2 discharges.

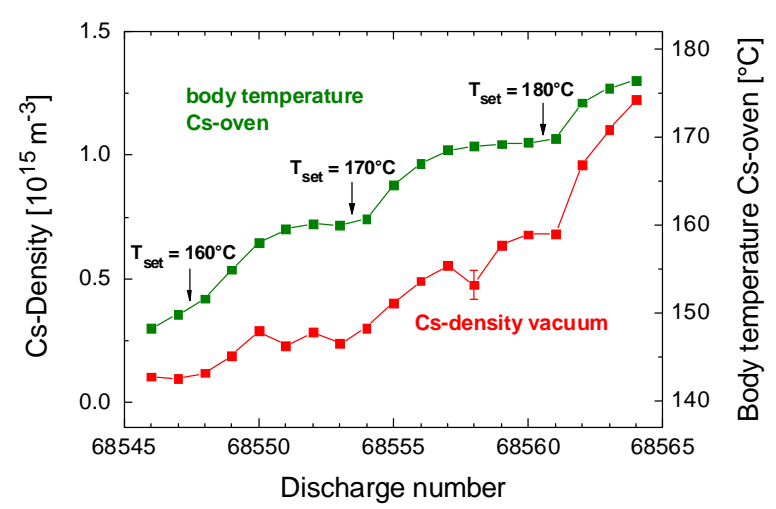

Figure 10. Growth of the caesium density in the vacuum phase and temperature of the cold spot of the caesium oven as a function of the discharge number of the negative ion test facility BATMAN (6 s discharge every $3 \mathrm{~min}$ ). 
Although the caesium oven is mounted at the backplate of the source body, the caesium density close to the plasma grid rises accordingly. Thus, the caesium distribution in the vacuum phase together with its redistribution by the plasma results in a fast response of the caesium density close to the grid. The latter is mandatory for supplying the plasma grid with caesium. Additionally, figure 10 shows that with higher set-value of the oven temperature the increase in the density is more pronounced and more time is needed until an equilibrium density is achieved, which is a typical behaviour as seen in many other measurements. This, in turn, is important for avoiding caesium accumulation in the source and thus unstable conditions. It is worth to notice that the sensitivity of the laser absorption is exactly in the required range and that already these first tests of measurements during caesium evaporation give some insight in the evaporation and distribution of caesium which was not measurable before and thus point of speculation. In addition, caesium evaporation of the oven can be determined by measuring the amount of caesium in vacuum and plasma operation and in turn the absorption signal might be usable as a control loop signal for caesium evaporation in future experiments.

Obviously, in the beginning of an experimental day the caesium amount in the volume above the grid is determined by the caesium evaporation. During the day, other effects like redistribution by the plasma and release from the plasma grid surface as well as surface temperatures of the source body and the grid might determine the caesium density in this particular volume.

The automatic data acquisition and analysis together with the coupling of the trigger of BATMAN allows for routine measurements, before, during, and after the discharge. Such a time trace is shown in figure 11 for one of the discharges plotted in figure 10. In the vacuum phase the caesium density is close to $10^{15} \mathrm{~m}^{-3}$. As the rf power is switched on a small peak or disturbance occurs until a more or less stable caesium density is been detected. The disturbance at the beginning of the discharge is caused by a change in the pressure due to a gas pulse which is commonly used at the IPP ion source to ensure plasma ignition.

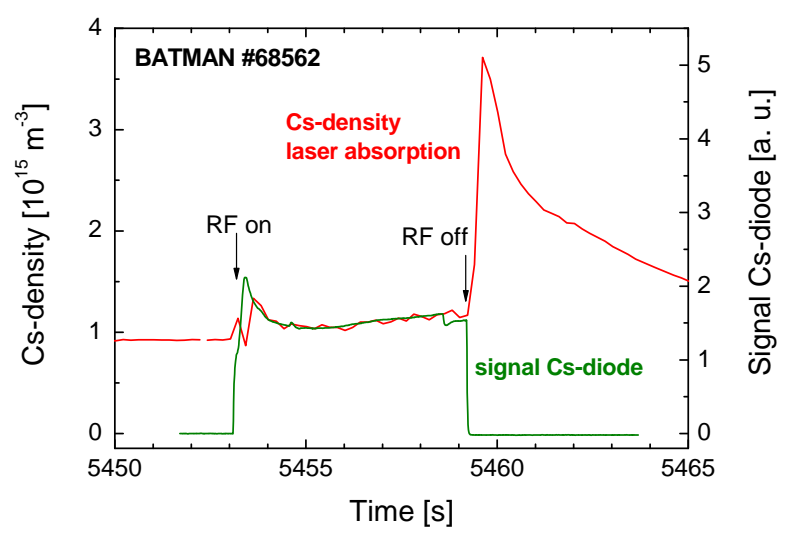

Figure 11. Typical time traces of the caesium density determined by laser absorption $3 \mathrm{~s}$ before the discharge is turned on, during the discharge ( $r f$ on and rf off) and $5 \mathrm{~s}$ after the discharge (0.6 Pa, $60 \mathrm{~kW}$, hydrogen).

During the discharge the density of neutral caesium atoms remains at a similar level as before which is quite surprising. At the plasma conditions of the ion source, i.e. electron densities in the range of $10^{17}$ $\mathrm{m}^{-3}$ and temperatures of $1 \mathrm{eV}$, above $90 \%$ of the caesium is ionized [18]. This means, starting with the vacuum phase a strong decrease of the signal is been expected. The non-appearance of the behaviour let to the conclusion that a strong redistribution of caesium from the surfaces appears during the discharge. As also shown in figure 11 is the OES signal obtained by a simple photodiode equipped with a interference filter with central wavelength of the caesium $852 \mathrm{~nm}$ line. Since the photodiode is 
not calibrated, the scaling of its signal is set to fit the measured Cs-density of the laser absorption spectroscopy. The comparison shows an excellent agreement in the temporal behaviour. As stated from earlier OES measurements the caesium density (neutrals) is mentioned to be in the range of $10^{15}$ $\mathrm{m}^{-3}[18,19]$, which of course depend on the caesium conditions but also on the knowledge of the exact plasma parameters, electron density and temperature. As discussed for the results obtained in the laboratory experiments, the laser absorption is the more reliable method.

After the rf power is been switched off the caesium density increases rapidly which is attributed to the recombination of caesium ions into neutrals. Thus, the ionization degree of caesium might be deduced from the peak height. However, recombination takes place in a much shorter time scale than can be measured by the laser absorption, which means the actual height of the peak is, for the present time resolution given, point of speculation. Nevertheless, a lower limit for the ionization can be deduced from the measure peak height which is determined to be roughly $70 \%$. The slow decay after the discharge reflects the diffusion time and sticking coefficient of the caesium particles towards and at the surfaces. For this example a decay time of $2 \mathrm{~s}$ is obtained.

In summary, it can be stated that these first examples demonstrated the applicability of laser absorption as a standard method for ion sources with first promising results giving already some new, quite surprising, insight into the caesium dynamics. The method itself was for the first time established at caesium-seeded hydrogen ion sources and opens a window for more systematic investigations of the caesium dynamic which will be described in a following paper.

\section{Conclusion}

The setup for the laser absorption diagnostics of caesium densities has been thoroughly tested in the laboratory before applying to the negative hydrogen ion sources which are in routine operation. The detection limits, the effect of line saturation and depletion are been discussed in detail and accounted for. The system has been optimized to operate under ion source conditions, which means being sensitive in the required density range and being equipped with an automatic data acquisition and analysis system. A temporal resolution of $40 \mathrm{~ms}$ is achieved; signal averaging reduces the time resolution but improves the signal-to-noise-ratio. The method is calibrated by using a caesium absorption cell with a well defined cold spot. Dedicated measurements and comparison with other techniques for caesium detection in a laboratory plasma chamber demonstrated applicability and excellent reliability of the method.

The usage at the IPP prototype rf ion source for ITER demonstrated clearly the applicability of the method in routine operation. The easy-to-use setup with its perfect dynamic range and a high signalto-noise ratio is perfectly suited for the needs of the $\mathrm{rf}$ ion source. First results gave already an insight into the dynamics of the caesium evaporation and the temporal behaviour of caesium before during and after a discharge. The method itself complements other diagnostic methods as the OES and does not rely on the knowledge of plasma parameters. However, the line-of-sight integrated caesium densities can be obtained only and a improved temporal resolution would be desirable to obtain the dynamics immediately after a discharge in more detail. The laser absorption has a high potential for bringing more insight into the caesium dynamics and thus further improvements of the ion sources with respect to faster source conditioning, reproducibility and reliability. It also has been demonstrated that the white light absorption spectroscopy works well in the small scale lab experiment with the possibility of an easy adaption to small sources for the determination of caesium dynamics. Further systematic studies of the laser absorption spectroscopy at the short pulse test facility BATMAN are underway and will be described in a subsequent paper. It is also planned to transfer the system to the long pulse test facility MANITU at IPP, which is capable to extract a beam of up to one hour. Of particular interest is the caesium dynamics during long pulse operation with short time slots between the pulses only. 


\section{Acknowledgement}

The work was partly supported by a grant from Fusion for Energy (F4E-2008-GRT-07). The opinions expressed herein are those of the authors only and do not represent the Fusion for Energy's official position. Financial support from ITER is also gratefully acknowledged.

\section{References:}

[1] Belchenko Y, Dimov G, and Dudnikov V 1974 Nucl. Fusion 14113

[2] Dudnikov V 2002 Rev. Sci. Instrum. 73992

[3] Van Amersfoort P W, Geerlings J J C, Kwakman L F Tz, et al. 1985 J. Appl. Phys. 583566

[4] Lee B S and Seidl M 1992 Appl. Phys. Letters 612857

[5] Boutry G A and Dormont H 1969 Philips Tech. Rev. 30225

[6] Graham W G, 1980 Proc. $10^{\text {th }}$ Symp. on Fus. Tech., Brookhaven National Laboratory, Upton

[7] Schletti R, Wurz P and Fröhlich T 2000 Rev. Sci. Instrum. 71499

[8] Gutser R, Wimmer C, and Fantz U 2011 Rev. Sci. Instrum. 82023506

[9] Peters J 2000 Rev. Sci. Instrum. 711069

[10] Leung K N, DeVries G J, DiVergilio D F et al. 1991 Rev. Sci. Instrum. 62100

[11] Rouleau G, Geros E, Stelzer J et al. 2008 AIP Conference Proceedings 79 p. 02A514

[12] Ikeda Y et al. 2006 Nucl. Fusion 46 S211

[13] Takeiri Y et al. 2006 Nucl. Fusion 46 S199

[14] http://www.iter.org

[15] Hemsworth R et al. 2009 Nucl. Fusion 49045006

[16] Speth E et al. 2006 Nucl. Fusion 46 S220

[17] Hemsworth R, Tanga A, and Antoni V 2008 Rev. Sci. Instrum. $7902 \mathrm{C} 109$

[18] Fantz U et al. 2005 Fusion Eng. Design. 74299

[19] Fantz U et al. 2009 Nucl. Fusion 49 (2009) 125007

[20] Fantz U, Gutser R, and Wimmer C 2010 Rev. Sci. Instrum. $8102 \mathrm{~B} 102$

[21] Sansonetti J E 2009 J. Phys. Chem. Ref. Data 38761

[22] Buuron A J M, Otorbaev D K, van de Sanden M C M, and Schram D C 1995 Rev. Sci. Instrum. 66968

[23] Bol'shakov AA and Cruden B A 2008 Technical Physics 531423

[24] Ralchenko Y, Kramida A E, Reader J, and NIST ASD Team 2010 NIST Atomic Spectra Database (ver. 4.0.1) available: http://physics.nist.gov/asd [2010, December 29].

[25] McNeely P and Wünderlich D 2011 Plasma Sources Sci. Technol. 2004005

[26] Steck D A: "Cesium D Line Data", available online at http://steck.us/alkalidata (revision 2.1.2) 2009

[27] White H E and Eliason A Y 1933 Phys. Rev. 44753

[28] Buuron A J M, Otorbaev D K, van de Sanden M C M, and Schram D C 1994 Phys. Rev. E 50 1383

[29] Booth J-P, Cunge G, Neuilly F, and Sadeghi N 1998 Plasma Sources Sci. Technol. 7423

[30] Condon E U 1964 PNAS 52635

[31] Sagle J, Namiotka K, and Huennekens J 1996 J. Phys. B 292629

[32] Fantz U, Wimmer C, AIP Conference Proceedings (NIBS2010) accepted

[33] Alcock, C B, Itkin, V P, and Horrigan, M K, 1984 Canadian Metallurgical Quarterly 23309

[34] Scheubert P, Fantz U, Awakowicz P and Paulin H, 2001 J. Appl. Phys. 90587

[35] SAES Getters S.p.A. Viale Italia 77, 20020 Lainate (Milan), Italy; http://www.saesgetters.com 
[36] Alvatec Production and Sales GesmbH, Eisenstrasse 62, 9330 Althofen, Austria; http://www.alvatec.com

[37] Christ Koch S et al., 2009 Plasma Sources Sci. Technol. 1802500

[38] McNeely P et al., Plasma Sources Sci. Technol. 18014011 